\title{
Serotyping group B streptococci in a small community hospital: an analysis of distribution and site of isolation
}

\author{
Jennifer M. Smith ${ }^{1,2, *}$, Jason A. Rexroth ${ }^{2, *}$, David G. Chaffin ${ }^{2}$ and \\ Susan H. Jackman ${ }^{1,2}$ \\ ${ }^{1}$ Department of Microbiology, Immunology and Molecular Genetics and \\ ${ }^{2}$ Department of Obstetrics and Gynecology, Joan C. Edwards School of Medicine, Marshall University, \\ Huntington, $W V$
}

\begin{abstract}
Objective: To determine the prevalence and site of isolation of different serotypes of group B streptococcus (GBS) colonization or infection at a small community hospital.

Methods: GBS isolates were obtained from a small community hospital and were then serotyped as la, Ib, II, III, IV, $V$ or nontypeable. Hospital records were reviewed for patient sex, age and pregnancy status as well as the site of GBS isolation.

Results: GBS serotypes la, III and V were most common and accounted for over $60 \%$ of the total number of isolates. Serotype la was most prevalent in reproductive-age females, while serotypes $V$ and III were most prevalent in non-reproductive-age females and males, respectively. Serotype la was most frequent in both pregnant and nonpregnant females. Serotype IV was more common in this study population than in those from other locations.

Conclusions: The GBS serotype distribution in this small community did not differ significantly from distributions described in larger North American centers. A GBS vaccine designed against multiple serotypes would be protective for most of this population.
\end{abstract}

Key words: Agglutination; Distribution; Vaccine; Group B Streptococcus

\section{INTRODUCTION}

Group B streptococcus (GBS) is the primary bacterial agent causing neonatal infections, including pneumonia, meningitis and sepsis. This organism has also been implicated in adult disease including postpartum infections, urinary tract infections, soft tissue infections and pneumonia ${ }^{1}$. While prophylactic antibiotic treatment implemented by many obstetricians has resulted in a significant reduction in infant morbidity and mortality, it does not address protection of the infant during the first critical months after birth or prevention of adult GBS disease. Vaccine development resulting in the generation of GBS-specific antibody is the strategy of choice. For effective vaccine design, identification of the most prevalent serotypes isolated from the general population is required. In the present study we determined the prevalence of GBS serotypes in a community hospital and compared these data to studies from larger centers in North America.

Correspondence to: Susan H. Jackman, PhD, Department of Microbiology, Immunology and Molecular Genetics, Joan C. Edwards School of Medicine, Marshall University, 1542 Spring Valley Drive, Huntington, WV 25704. Email: Jackman@marshall.edu

*These authors have contributed equally to this study. 


\section{SUBJECTS AND METHODS}

\section{Study population}

Medical records were reviewed from 107 patients who were identified as GBS-culture-positive by the Cabell Huntington Hospital microbiology laboratory (Huntington, WV) from August to November 1999. Patients were classified by retrospective chart review according to sex, age, pregnancy status, previous positive GBS culture and the site of GBS isolation. One hundred and two of the isolates were from females while only five isolates were from males. Patients ranged in age from 18 months to 92 years old. Nonreproductive age females were defined as 18 months to 13 years of age and from 40-92 years of age. Information on race for this population was not available; however, the majority of the communities that are served by this hospital are Caucasian. The most common site of isolation was from genital or urine cultures of reproductive-age females. GBS was also isolated from nonurogenital surfaces including a foot wound, endotracheal tube secretions, blood, a peritonsilar abscess and throat cultures.

\section{GBS isolation}

Bacterial cultures obtained from the hospital were streaked on $5 \%$ sheep blood tryptic soy agar plates and incubated overnight at $37^{\circ} \mathrm{C}$ in $5 \% \mathrm{CO}_{2}$ to establish a pure GBS culture. A single colony from each plate was inoculated into Todd Hewitt broth (THB) and incubated overnight at $37^{\circ} \mathrm{C}$ in $5 \%$ $\mathrm{CO}_{2}$. The overnight cultures were mixed 1:1 (volume:volume) with $24 \%$ sterile glycerol and frozen at $-20^{\circ} \mathrm{C}$ until use.

\section{GBS serotype determination}

GBS were serotyped using a hemolytic streptococcus group B typing sera kit (Accurate Chemical \& Scientific Corporation, Westbury, NY) according to manufacturer's instructions. Briefly, $10 \mu \mathrm{l}$ of GBS culture from frozen stocks were inoculated into $5 \mathrm{ml} \mathrm{THB}$ and incubated overnight at $37^{\circ} \mathrm{C}$ in $5 \% \mathrm{CO}_{2}$. The overnight cultures were centrifuged at $1400 \times g$ for 20 minutes. After discarding the supernatant, the GBS pellet was suspended in $0.5 \mathrm{ml}$ THB. Digestive enzymes from swine pancreas extracts were added to the GBS suspension prior to incubation at $37^{\circ} \mathrm{C}$ for 1 hour. The GBS suspension was centrifuged at $1400 \times g$ for 20 minutes. The bacterial pellet was suspended in $0.5 \mathrm{ml}$ phosphate-buffered saline (PBS) and incubated at $120^{\circ} \mathrm{C}$ for 30 minutes. Serotyping of the GBS extract was accomplished by agglutination with serotype-specific antisera (Ia, Ib, II, III, IV or V). PBS was used in place of the antisera as an agglutination control. Agglutination as noted visually or by dark field microscopy was considered a positive serotype result for that isolate. Any extract that failed to agglutinate or demonstrated agglutination in the PBS control was repeated. GBS isolates were labeled as nontypeable (NT) if they failed to agglutinate with any of the antisera or repeatedly agglutinated in all antisera and the PBS control.

\section{Statistical analysis}

Statistical analysis was performed using SigmaStat (Jandel Scientific, San Rafael, CA). Chi-square was used for comparisons of serotype frequency between reproductive and non-reproductive-age females, pregnant and nonpregnant females, isolation from the vagina versus the urine and females with vaginitis versus females with no symptoms.

\section{RESULTS}

One hundred and seven GBS isolates were obtained from patients at a community hospital in Huntington, WV. The GBS serotype frequencies categorized by patient sex and age, as well as isolation from the vagina or urine, are shown in Table 1. Serotypes Ia, V and III were the most common in this population with frequencies of 23, 21 and 20\% respectively. Serotype IV was the least frequent, accounting for only $5 \%$ of the total number of GBS isolates. Nine percent of the isolates were nontypeable by agglutination. Restriction fragment length polymorphism analysis on the capsule locus, as described previously by Sellin and colleagues ${ }^{2}$, was attempted to assign serotypes to the NT isolates. However, this method failed to provide any additional information (data not shown). 
Table I GBS serotypes by patients' sex, age and site of isolation

\begin{tabular}{|c|c|c|c|c|c|c|c|}
\hline \multirow[b]{3}{*}{ Serotype } & \multirow[b]{3}{*}{ Males } & \multicolumn{5}{|c|}{ Females } & \multirow[b]{3}{*}{ Percent of total } \\
\hline & & \multicolumn{3}{|c|}{ Age (years) } & \multicolumn{2}{|c|}{ Isolation site } & \\
\hline & & $<13$ & $13-39$ & $>39$ & Vagina* & Urine** & \\
\hline la & I & 0 & 21 & 3 & 19 & 5 & 23 \\
\hline $\mathrm{lb}$ & I & 2 & II & $\mathrm{I}$ & 13 & I & 14 \\
\hline II & 0 & 0 & 8 & I & 9 & 0 & 8 \\
\hline III & 2 & 2 & 15 & 2 & 15 & 3 & 20 \\
\hline IV & 0 & 0 & 4 & $\mathrm{I}$ & 4 & I & 5 \\
\hline V & I & $\mathrm{I}$ & 14 & 6 & 15 & 4 & 21 \\
\hline NT & 0 & 0 & 5 & 5 & 9 & I & 9 \\
\hline
\end{tabular}

*GBS were isolates from swabs taken from the lower third of the vagina and the rectum for pregnant females, while swabs were taken from the vagina only for symptomatic, nonpregnant women; **urine specimens were considered positive for GBS when > 10000 colony-forming units were present; NT, nontypeable

The majority of isolates were obtained from reproductive-age women $(73 \%)$ while males accounted for only $5 \%$ of the total. In the male population, serotype III was most commonly isolated. No statistical comparisons between the female and male serotype distributions were made due to the small number of GBS isolates from males $(n=5)$. In reproductive-age females, serotype Ia was the most frequent, while serotype $\mathrm{V}$ was most frequent in non-reproductive-age females. However, the individual serotype frequencies between these two groups were not significantly different $(p=0.273)$ (Table 1). Additional comparisons made with respect to pregnancy status $(p=0.239)$, site of isolation (vaginal versus urine; $p=0.71$ ), and symptomatic vaginitis (versus asymptomatic females; $p=0.511$ ) revealed no significant differences.

Eleven percent of the women in this study had infants with neonatal intensive care unit visits. None of these infants was under observation for GBS infection. All six GBS serotypes were isolated from these women; however, serotypes Ib, II and $\mathrm{V}$ were most frequent with three isolates each. In addition, the five females with previous positive GBS cultures were also carrying isolates of the most common serotypes: Ia $(n=2)$; III $(n=1)$; and $\mathrm{V}(n=2)$.

\section{DISCUSSION}

It has been well documented that antibodies to GBS-serotype-specific capsule polysaccharide are protective ${ }^{3,4}$. Unfortunately, few individuals generate sufficient quantities of these antibodies. Recent attempts at designing a vaccine against serotype III GBS yielded less than ideal results with only $40 \%$ of women vaccinated generating antitype III antibodies ${ }^{5}$. Protein carriers, including the tetanus toxoid ${ }^{6}$, are being evaluated for their ability to enhance the immunogenicity of the GBS capsule polysaccharide. Although such a protein-polysaccharide conjugate vaccine shows promise, a vaccine against multiple GBS serotypes will be most efficacious. Harrison and colleagues ${ }^{2}$ report that a pentavalent vaccine against serotypes Ia, Ib, II, III and V would protect approximately $95 \%$ of their study population.

The optimal design for a multivalent GBS vaccine will include the most common capsule polysaccharide serotypes. To date, much of the data regarding GBS serotype distribution has been obtained from large centers. The purpose of our study was to determine the serotype frequencies in a small community hospital in West Virginia and to compare our data to those from larger North American centers. 
Table 2 Comparison of GBS serotype frequencies between Huntington, WV and other North American populations

\begin{tabular}{|c|c|c|c|c|c|c|}
\hline \multirow[b]{2}{*}{ Serotype } & \multicolumn{6}{|c|}{ Positive GBS frequency (percent) } \\
\hline & WV & $M D^{7, *}$ & $I A^{8}$ & $T X^{9, * *}$ & Alberta ${ }^{10}$ & Calgary"I \\
\hline la & 23 & 28 & 25 & 24 & 17 & 22.7 \\
\hline $\mathrm{lb}$ & 14 & 12 & 6 & 9 & 10 & 10.7 \\
\hline ॥ & 9 & 12 & 5 & 26 & 9 & 12.9 \\
\hline III & 20 & 16 & 52 & 24 & 19 & 20.6 \\
\hline IV & 5 & $<1$ & 0 & 0 & 0 & 2.1 \\
\hline V & 21 & 26 & 9 & 12 & 31 & 19.3 \\
\hline NT & 9 & 4 & 3 & 4 & 13 & 11.2 \\
\hline
\end{tabular}

*Values represent data on adults compiled from Table I in Harrison et al ; **Values represent data compiled from Table 2 in Hickman et al'; WV, West Viginia; MD, Maryland; IA, lowa; TX, Texas; NT, nontypeable

Comparison of the West Virginia serotype frequencies with those observed primarily in adults or pregnant women from Maryland ${ }^{7}$; Iowa ${ }^{8}$; Texas ${ }^{9}$; Alberta, Canada ${ }^{10}$; and Calgary, Canada ${ }^{11}$ is shown in Table 2. The three most common serotypes in our study and the others are Ia, III and $\mathrm{V}$ with the exception of Texas where Ia, II and III were the most prevalent. While serotype IV was least common in all populations, in our study it accounted for $5 \%$ of the total number of isolates, which differs from the other study populations that report frequencies of $0-2 \%$. Although this difference is slight, it might indicate that serotype IV GBS is an emerging serotype in our community. This trend should be monitored in the future as a shift in the serotype distributions may alter efficacy of a potential vaccine. Nontypeable isolate frequencies ranged from 3 to $13 \%$ in the large North American centers. Our West Virginia population was $9 \%$ nontypeable.
Of the centers with which we compared our data, our population most closely resembles those from Canada. Yet overall the serotype distribution in our West Virginia community does not differ greatly from any of the larger centers. As such, the pentavalent vaccine proposed by Harrison ${ }^{2}$ would be effective in protecting at least $86 \%$ of our population. Based on this study, we are confident that our community is similar enough to large metropolitan areas that a multivalent vaccine designed from serotype frequencies in these communities would also be effective for our area.

\section{ACKNOWLEDGEMENTS}

We thank Constance M. Berk for her technical assistance and the staff of the Cabell Huntington Hospital clinical microbiology laboratory for their assistance in obtaining the GBS specimens.

\section{REFERENCES}

1. Schuchat A. Epidemiology of group B streptococcal disease in the United States: shifting paradigms. Clin Microbiol Rev 1998;11:497-513

2. Sellin M, Olofsson C, Hakansson S, Norgren M. Genotyping of the capsule gene cluster (cps) in nontypeable group B streptococci reveals two major cps allelic variants of serotypes III and VII. J Clin Microbiol 2000;38:3420-8
3. Shigeoka AO, Hall RT, Hemming VG, et al. Role of antibody and complement in opsonization of group B streptococci. Infect Immun 1978;21:34-40

4. Kallman J, Schollin J, Schalen C, et al. Impaired phagocytosis and opsonization towards group B streptococci in preterm neonates. Arch Dis Child Fetal Neonatal Ed 1998;78:F46-50

5. Baker CJ, Rench MA, Edwards MS, et al. Immunization of pregnant women with a polysaccharide 
vaccine of group B streptococcus. $N$ Engl J Med 1988;319:1180-5

6. Kasper DL, Paoletti LC, Wessels MR, et al. Immune response to type III group B streptococcal polysaccharide-tetanus toxoid conjugate vaccine. J Clin Invest 1996;98:2308-14

7. Harrison LH, Elliot JA, Dwyer DM, et al. Serotype distribution of invasive group B streptococcal isolates in Maryland: implications for vaccine formulation. J Infect Dis 1998;177:998-1002

8. Andrews JI, Diekema DJ, Hunter SK, et al. Group B streptococci causing neonatal bloodstream infection: antimicrobial susceptibility and serotyping results from SENTRY centers in the western hemisphere. Am J Obstet Gynecol2000;183:859-62

ReCEIVED 02/12/02; ACCEPTED 04/19/02
9. Hickman ME, Rench MA, Ferrieri P, Baker CJ. Changing epidemiology of group B streptococcal colonization. Pediatrics 1999;104:203-9

10. Tyrrell GJ, Senzilet LD, Spika JS, et al. Invasive disease due to group B streptococcal infection in adults: results from a Canadian, population-based, active laboratory surveillance study - 1996. J Infect Dis 2000;182:168-73

11. Davies HD, Adair C, McGreer A, et al. Antibodies to capsular polysaccharides of group B Streptococcus in pregnant Canadian women: relationship to colonization status and infection in the neonate. J Infect Dis 2001;184:285-91 


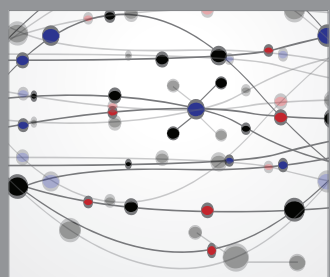

The Scientific World Journal
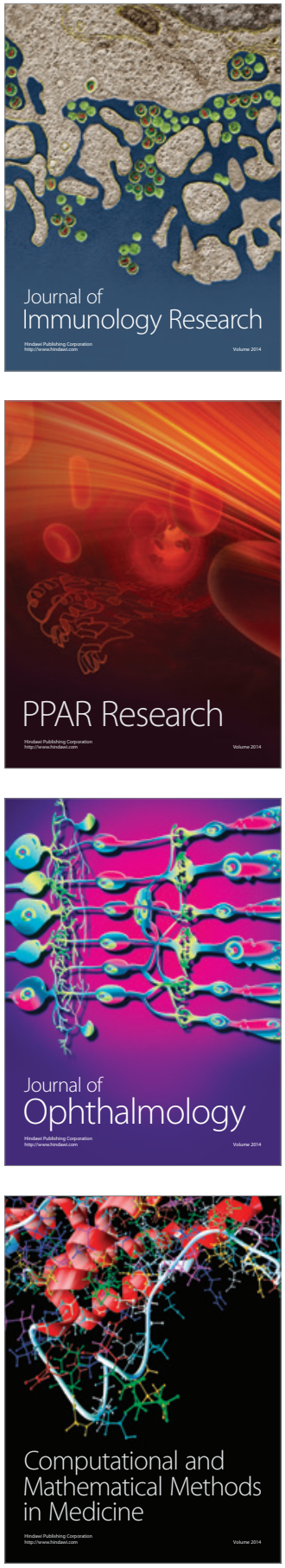

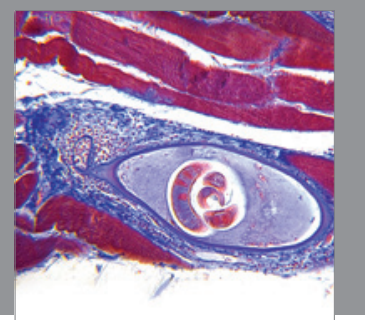

Gastroenterology

Research and Practice
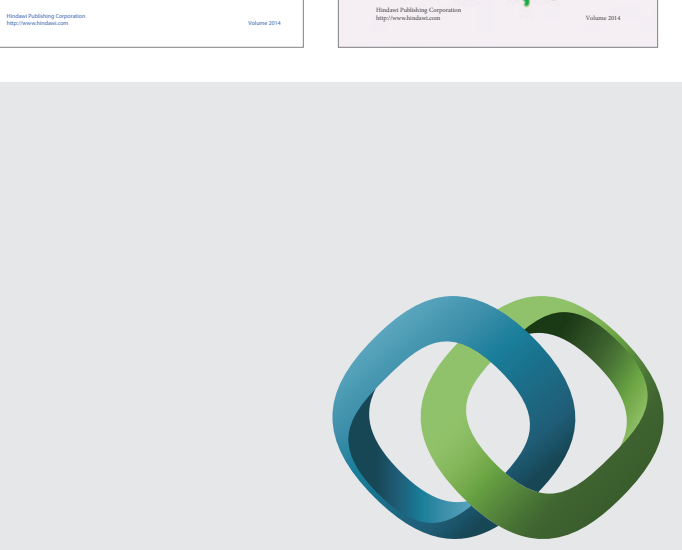

\section{Hindawi}

Submit your manuscripts at

http://www.hindawi.com
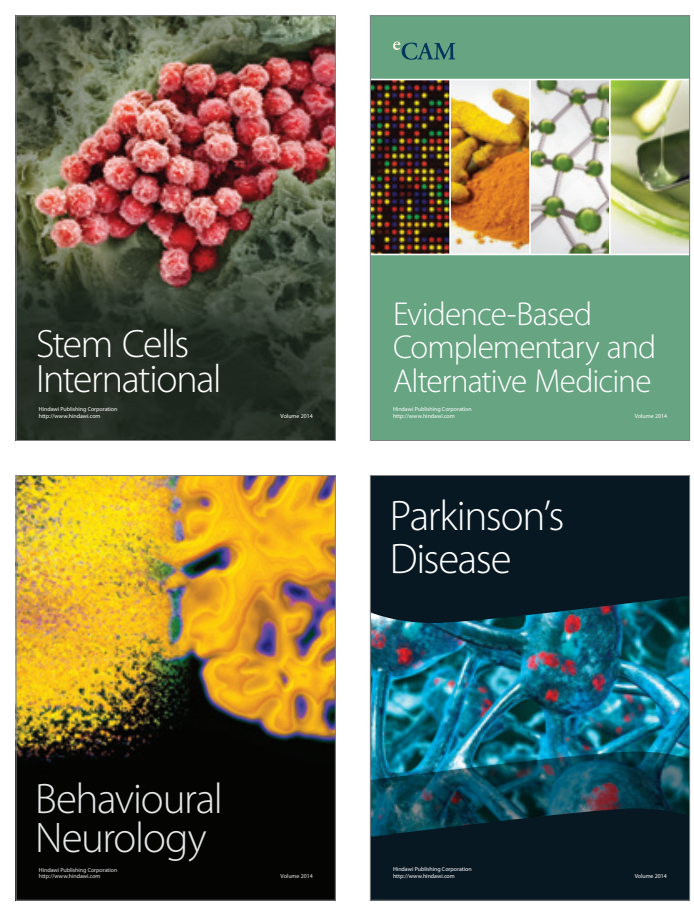

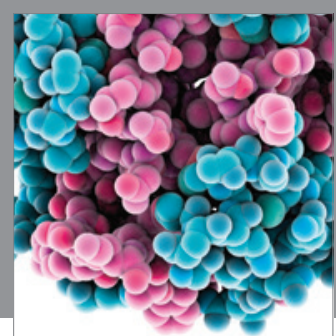

Journal of
Diabetes Research

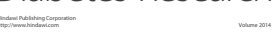

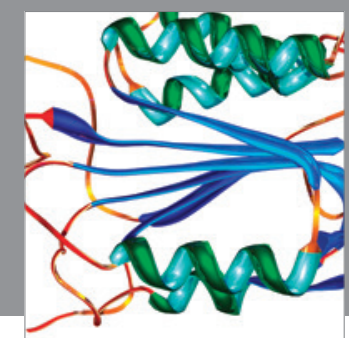

Disease Markers
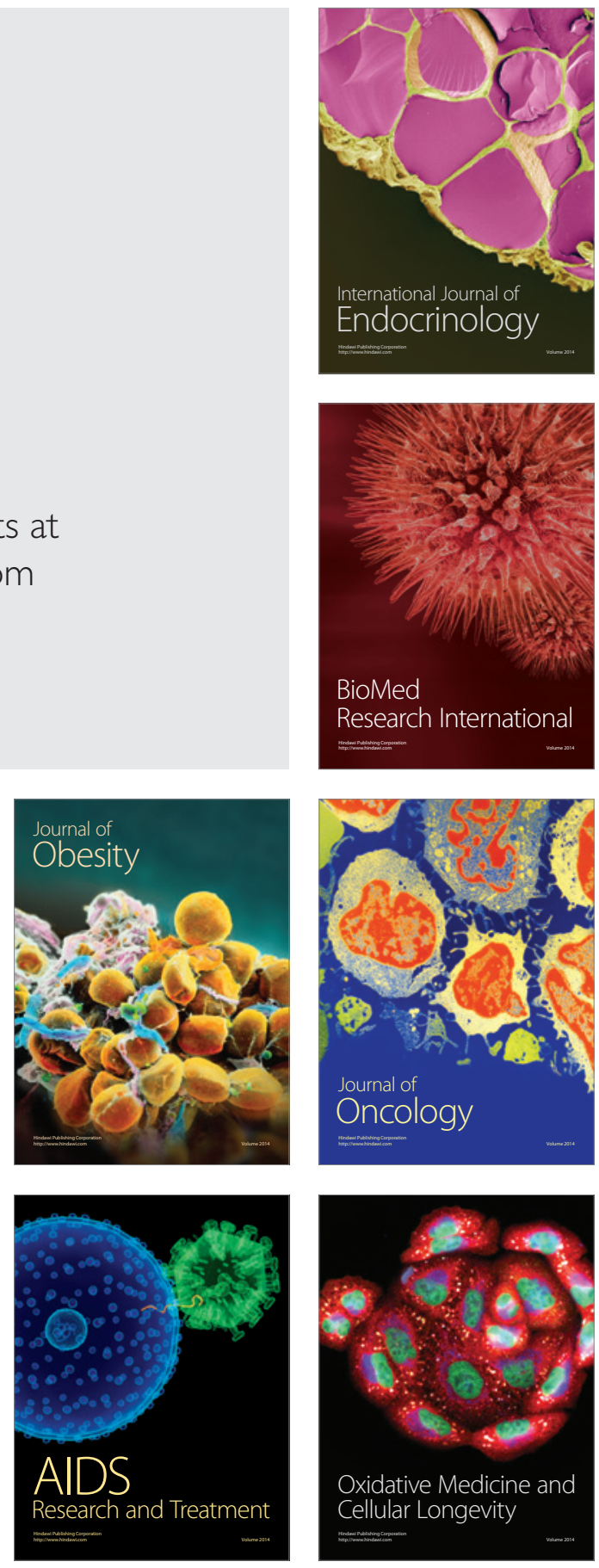